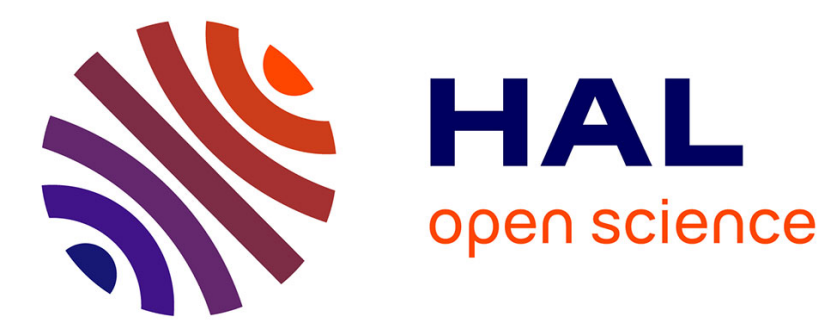

\title{
Game theory approach for modeling competition over visibility on social networks
}

Alexandre Reiffers-Masson, Yezekael Hayel, Eitan Altman

\section{To cite this version:}

Alexandre Reiffers-Masson, Yezekael Hayel, Eitan Altman. Game theory approach for modeling competition over visibility on social networks. Social Networking Workshop (in conjunction with COMSNETS 2014), Jan 2014, Bangalore, India. pp.1-6, 10.1109/COMSNETS.2014.6734939 . hal00918036v2

\section{HAL Id: hal-00918036 https://hal.inria.fr/hal-00918036v2}

Submitted on 28 Mar 2016

HAL is a multi-disciplinary open access archive for the deposit and dissemination of scientific research documents, whether they are published or not. The documents may come from teaching and research institutions in France or abroad, or from public or private research centers.
L'archive ouverte pluridisciplinaire HAL, est destinée au dépôt et à la diffusion de documents scientifiques de niveau recherche, publiés ou non, émanant des établissements d'enseignement et de recherche français ou étrangers, des laboratoires publics ou privés. 


\title{
Game theory approach for modeling competition over visibility on social networks
}

\author{
Alexandre Reiffers Masson, Yezekael Hayel, Eitan Altman
}

September 27, 2015

\begin{abstract}
In Online Social Networks, such as Facebook, Linkedin, Twitter, Google+ and others, many members post messages to walls or to timelines of their friends. There is a permanent competition over content visibility since timelines have finite capacity. As new content arrives, older content gets pushed away from the timeline. A selfish source that wishes to be visible has to send from time to time new content thus pre-empting other content from the timeline. We assume that sending more content comes with some extra cost. We study the problem of selecting the rates of content creation as a non-cooperative game between several sources that share some common destination to which they send content. We identify conditions under which the problem can be reduced to the Kelly mechanism for which we compute explicitly the equilibrium. This is done in a very general probabilistic framework where time between arrivals of content is only required to be stationary ergodic.
\end{abstract}

\section{Introduction}

The visibility of contents in timelines is limited due to capacity limits of the timeline. It is also impacted by visibility restriction due to a finite screen size and by the scrolling habits of users. Sources of content that wish to be visible on a given timeline have to take into account other flows of content that compete over space in the same timeline. Thus they have incentives to resend copy of their contents in order to make their opinions visible. In absence of 
such actions, the average number of their posts seen in the Timeline may be very small. The choice of rates of content can be formulated as a noncooperative game where a source's utility is the difference between some visibility utility and some cost for sending and creating contents.

In a previous paper, the authors [12] studied a similar game theory model under the following assumptions:

- The arrival process of contents is Poisson distributed;

- The visibility utility of a source is assumed to be the indicator of having at least one content of that source in the timeline.

In this paper we abandon the restrictive Poisson assumption. We allow general dependence of times between contents arrival. We shall only require that the arrival time of content for each different source $i$ is a stationary ergodic point process, and that their superposition is a simple process (at a given time there may only be a single content arrival).

The assumption of stationary behaviour is well suited to a subscriber's wall. Indeed, there is no reason to expect that the user will access to his timeline at times with a distribution, which is not time-uniform. We assume that a user does not observe the system at a stationary regime but rather at instants of arrivals of new contents. This hypothesis is coming from the fact that we assume that users receive notifications whenever a new content arrives. In the theory of point process, the system observed at arrival instants of points in a stationary ergodic point process corresponds to the so-called Palm probability.

Another difference with respect to [12] is that we shall consider in this paper the total number of contents at arrival times of contents as the visibility payoff.

Our first result of this paper is to show that the problem can be reduced to the well known generalized Kelly mechanism, which allows us to use many existing results to characterize the equilibrium. Moreover we derive the equilibrium in explicit form and thus obtain new results for the generalized Kelly mechanism. We then extend the model to study rerouting of posts, which corresponds to sharing content. 


\section{Related Work}

We briefly discuss the generalised Kelly mechanism [15]. Consider a resource $K$ that is to be shared among $J$ strategic users. A user proposes a bid $\lambda_{j} \in[0, \bar{\lambda}]$. Then the resource is shared proportionally to the bids, so that the amount of resource that player $j$ receives is

$$
K \frac{\lambda_{j}}{\sum_{i} \lambda_{i}}
$$

and each player $j$ has a linear cost $\gamma$ for his bid. Finally his objective function is:

$$
U_{j}\left(K \frac{\lambda_{j}}{\sum_{i} \lambda_{i}}\right)-\gamma \lambda_{j}
$$

where $U_{j}($.$) is a function that measures the satisfaction that receive the player$

$j$ when he has $K \frac{\lambda_{j}}{\sum_{i} \lambda_{i}}$ of the resource. A study of this game was proposed in [6]. Some networking applications of this game can be found in [2] and [3].

A similar analytical study works like ours has been recently proposed in [12]. In this paper, the authors propose to study the competition over a timeline between several information providers (sources). There are several differences with our paper. Firstly, they consider Poisson arrival rates whereas, in our model, we consider a more general point process. Secondly, they define the visibility over a news feed as the proportion of time one message is visible on the timeline. Then, they consider in fact the case that only one message of each source can appear in the timeline. In our analysis we also generalize this point by considering that several messages, from the same source, can be visible on the timeline. Finally, we consider the propagation effect between news feed, which is not taking into account in [12].

\section{The game formulation}

Consider a single timeline of size $K$. It can contain up to $K$ posts. Consider an arrival of content to that timeline which is a superposition of $J$ stationary ergodic point processes $N_{j}(j=1, \ldots, N)$ assumed to be compatible with the flow $\theta_{t}$, each of which has a finite and non-null intensity $\lambda_{j}$. We call by $N$ their superposition. Each source $j$ is associated to the point process of intensity $\lambda_{j}$. We use the definition of [13] for the Palm probability which is the probability that a message is seen at an arrival instant. Let $P_{N}^{o}(A)$ be 
the Palm probability corresponding to the point process $N$, and we define similarly the Palm process that corresponds to point process $N_{j}$. It can be shown in page 37 or [13] that

$$
P_{N}^{o}\left(N_{j}(\{0\}=1)\right)=\frac{\lambda_{j}}{\sum_{i} \lambda_{i}}
$$

This formula states that when an arrival occurs, the probability that it is from source $j$ is $\frac{\lambda_{j}}{\sum_{i} \lambda_{i}}$.

We consider the dissemination utility for player $i$ to be the sum of messages originating from it in the timeline at an arbitrary arrival instant of a content. This event can be written as

$$
\sum_{j=0}^{K-1} 1\left(N_{j}^{o} T_{-j}=1\right)
$$

whose expectation with respect to the Palm probability measure is given by

$$
\sum_{j=0}^{K-1} P_{N}^{o}\left(N_{j} T_{-j}=1\right)=K \frac{\lambda_{j}}{\sum_{i} \lambda_{i}}
$$

due to (1.3.17) p 25 in [13] and eq (1).

We assume that each source $j$ has a cost for sending messages, which depends on his rate $\lambda_{j}$. This cost is expressed by $\gamma_{j} \lambda_{j}$. Finally, source's $j$ objective function is defined by the difference between the average number of messages and the cost associated to the creation of contents:

$$
U_{j}\left(\lambda_{j}, \lambda_{-\mathbf{j}}\right)=K \frac{\lambda_{j}}{\sum_{i} \lambda_{i}}-\gamma_{j} \lambda_{j}
$$

where $\lambda_{-\mathbf{j}}=\left\{\lambda_{1}, \ldots, \lambda_{j-1}, \lambda_{j+1}, \ldots, \lambda_{J}\right\}$ is the strategy vector of all the other sources.

\section{Competition between sources}

We are studying the visibility competition between the sources. We are studying a normal form non-cooperative game with $J$ sources. The utility function of each source depends of its own rate and the one of other sources. The utility function of source $j$ is given in equation (2). 
Definition 1. The decision vector $\lambda^{*}=\left(\lambda_{1}^{*}, \ldots, \lambda_{J}^{*}\right)$ is a Nash equilibrium if for all $j \in\{1, \ldots, J\}$,

$$
U_{j}\left(\lambda_{j}^{*}, \lambda_{-j}^{*}\right)=\max _{\lambda_{j} \in[0, \bar{\lambda}]} U_{j}\left(\lambda_{j}, \lambda_{-j}^{*}\right),
$$

where $\bar{\lambda}$ is the maximum source rate.

In other words, a source has not an interest in changing his rate unilaterally at the Nash equilibrium. This game was already originally proposed in [6] but never applied in the context of Online Social Networks. It has been also used to study competition over popularity in [1], but without considering a finite number of messages of interest in a Timeline. This game has several important properties. First, at the Nash equilibrium, at least one source send messages in the timeline as it is stated in the next proposition.

Proposition 2. The decision vector $(0, \ldots, 0)$ is not a Nash equilibrium.

Next in the following theorem, we generalize the results obtained in [1]. In this theorem, we obtain an explicit solution of the unique equilibrium of the game, when each source sends messages in the timeline.

Theorem 3. If $\sum_{i} \gamma_{i}-\max _{j}\left\{\gamma_{j}\right\}(J-1)>0$, the Nash equilibrium is uniquely defined by:

$$
\forall j, \quad \lambda_{j}^{*}=\frac{(J-1) K}{\sum_{i} \gamma_{i}}\left(1-\frac{\phi \gamma_{j}}{K}\right)>0 .
$$

with $\phi=\frac{(J-1) K}{\sum_{i} \gamma_{i}}$.

In the previous theorem, there is a condition over the cost $\gamma_{j}$ for all $j$ such that $\lambda_{j}>0$ for all $j$. If this condition is not satisfied, some rates can be equal to 0 at equilibrium for some sources. This result is described in the next theorem. Given the explicit expression of the Nash equilibrium of the game, we are able to understand better the behaviour of the equilibrium when the parameters of the game change. Thus from theorem 3 , it can be noticed that for all source $j$, the rate $\lambda_{j}^{*}$ is strictly decreasing in $\gamma_{j}$ and linear increasing in $K$. Thus we can now compute a general form of the equilibrium and not just restrict our self to an equilibrium where $\sum_{i} \gamma_{i}-\max _{i}\left\{\gamma_{j}\right\}(J-1)>0$, in other word when all $\lambda_{j}^{*}$ are positive. Assume we rearrange the sources, from smallest $\gamma_{j}$ to largest, then $\gamma_{1}>\gamma_{2}>\ldots>\gamma_{J}$. 
Theorem 4. Assume that $j^{\prime}$ is such that $j^{\prime}=\max \left\{j \mid \frac{(J-1) K}{\sum_{i=j}^{J} \gamma_{i}}\left(1-\frac{(J-1) \gamma_{j}}{\sum_{i=j}^{J} \gamma_{i}}\right) \leq\right.$ $0\}$ i.e $j^{\prime}$ is the source with largest $\gamma_{j}$ who doesn't send any messages in the timeline. In this case the Nash Equilibrium is uniquely defined by:

$$
\begin{array}{ll}
\lambda_{j}^{*}=\frac{\left(J^{\prime}-1\right) K}{\sum_{i>j^{\prime}} \gamma_{i}}\left(1-\frac{\left(J^{\prime}-1\right) \gamma_{j}}{\sum_{i>j^{\prime}}^{J} \gamma_{i}}\right) & \forall j>j^{\prime} \\
\lambda_{j}^{*}=0 & \forall j \leq j^{\prime}
\end{array}
$$

where $J^{\prime}=J-\left|\left\{1, \ldots, j^{\prime}\right\}\right|$.

In order to study the inefficiency of the Nash Equilibrium we study the price of anarchy [14]. We adopt the following assumption, for each $j, \lambda_{j} \in$ $[\underline{\lambda}, \bar{\lambda}]$, with $\underline{\lambda}>0$. First we define the social welfare of the game:

$$
V\left(\lambda_{1}, \ldots, \lambda_{J}\right)=\sum_{i}\left[K \frac{\lambda_{i}}{\sum_{i} \lambda_{i}}-\gamma_{j} \lambda_{i}\right]=K-\sum_{i} \gamma_{j} \lambda_{i}
$$

We maximize the social welfare:

$$
\left(\lambda_{1}^{\prime}, \ldots, \lambda_{J}^{\prime}\right)=\operatorname{argmax} V\left(\lambda_{1}, \ldots, \lambda_{J}\right)=(\underline{\lambda}, \ldots, \underline{\lambda})
$$

- The price of anarchy is define by:

$$
P_{O} A=\frac{V\left(\lambda_{1}^{\prime}, \ldots, \lambda_{J}^{\prime}\right)}{\sum_{j} U_{i}\left(\lambda_{j}^{*}, \lambda_{-j}^{*}\right)}
$$

Theorem 5. If $\sum_{i} \gamma_{i}-\max _{j}\left\{\gamma_{j}\right\}(J-1)>0$ then the price of anarchy is:

$$
P o A=\frac{\left(\sum_{i} \gamma_{i}\right)\left(K-\underline{\lambda} \sum_{i} \gamma_{i}\right)}{\sum_{j}\left(\sum_{i} \gamma_{i}-(J-1) \gamma_{j}\right)\left(\sum_{i} \gamma_{i}-\gamma_{j}(J-1) K\right)}
$$




\section{Propagation effect within 2 timelines}

We consider a generalization of our model to take into account the propagation effect between several timelines. In fact, some messages posted on one timeline can be relayed (propagated) to another timeline. A message which is of interest to one community can be of interest to another community for which the center of interest is closed. We are interested in one particular question for the sources:

Knowing the propagation effect between timelines, how will each source decide to send his information flow to the timelines, directly or indirectly using the propagation effect ?

Indeed, we have assumed that posting a message on a timeline has a cost for the source, whereas if a message is relayed, it is free for the source. Thus, in this section, we study this topological effect on the non-cooperative game between the sources. In order to get interesting closed-form results, we reduce the complexity of the game analysis by considering two timelines, $L=2$, but our results will be extended to more complex topologies in future works. First, in the next sub-section, we define the point process model the characterizes the arrival of the messages for the different timelines.

\subsection{Point process model}

Now assume that you have 2 timelines $l \in\{1,2\}$. Each source $j \in\{1, \ldots, J\}$ can send messages to each timeline 1 and 2 . It means that each source $j$ controls 2 point processes, which represent flow of messages of source $j$ in each timeline, with intensity $\lambda_{j}^{l}=E\left[N_{j}^{l}((0,1])\right]$ for $l=1,2$. For each timeline $l$, any new message will be copied and posted, with a probability $p_{l l^{\prime}}$, to the other timeline $l^{\prime} \neq l$. This process defines a new point process in each timeline $l$ with intensity $p_{l^{\prime} l} \sum_{j} \lambda_{j}^{l^{\prime}}$. If we add all the point processes for a timeline $l$ (direct and indirect messages), this new point process $N^{l}$ has an intensity $\sum_{i}\left(\lambda_{i}^{l}+p_{l^{\prime} l} \lambda_{i}^{l^{\prime}}\right)$. We assume that $K^{l}$ is the number of messages that subscribers of timeline $l$ take care of it. We denote by $T_{n}^{l}$ the arrival time of the $n^{\text {th }}$ message in the timeline $l$. Let $N_{j}^{l}\left(\left(T_{n}, T_{n+K^{l}}\right]\right)$ the number of messages from source $j$ in timeline $l$, as long as the $n^{\text {th }}$ message is visible on the timeline, i.e. is part of the first $K^{l}$ messages. Source $j$ wants to maximize $\bar{N}_{j}$ that is the expected number of messages from him that are visible on all 
the timelines:

$$
\bar{N}_{j}=\lim _{N^{\prime} \rightarrow+\infty} \sum_{l} \frac{1}{N^{\prime}} \sum_{n=1}^{N^{\prime}} N_{j}^{l}\left(\left(T_{n}^{l}, T_{n+K^{l}}^{l}\right]\right) .
$$

By applying the similar analysis as in section for IV, we obtain the following closed-form expression:

$$
\bar{N}_{j}=K^{1} \frac{\lambda_{j}^{1}+p_{21} \lambda_{j}^{2}}{\sum_{i}\left(\lambda_{i}^{1}+p_{21} \lambda_{i}^{2}\right)}+K^{2} \frac{\lambda_{j}^{2}+p_{12} \lambda_{j}^{1}}{\sum_{i}\left(\lambda_{i}^{2}+p_{12} \lambda_{i}^{1}\right)} .
$$

Let $\gamma_{j}^{l}$ be the cost from source $j$ for sending one message to timeline $l$. We denote the control vector of source $j$ by $\lambda_{\mathbf{j}}=\left\{\lambda_{j}^{1}, \lambda_{j}^{2}\right\}$. The objective function of each source $j$ is:

$$
\begin{aligned}
U_{j}\left(\lambda_{\mathbf{j}}, \lambda_{-\mathbf{j}}\right)= & K^{1} \frac{\lambda_{j}^{1}+p_{21} \lambda_{j}^{2}}{\sum_{i}\left(\lambda_{i}^{1}+p_{21} \lambda_{i}^{2}\right)}+K^{2} \frac{\lambda_{j}^{2}+p_{12} \lambda_{j}^{1}}{\sum_{i}\left(\lambda_{i}^{2}+p_{12} \lambda_{i}^{1}\right)} \\
& -\left(\gamma_{j}^{1} \lambda_{j}^{1}+\gamma_{j}^{2} \lambda_{j}^{2}\right) .
\end{aligned}
$$

\subsection{Game analysis}

For the analysis of (3), we consider another variable for the controls of the players, and then we define a new game where the objectif function for each player $j$ is:

$$
\max _{\Lambda_{j}^{1}, \Lambda_{j}^{2}} U_{j}\left(\boldsymbol{\Lambda}_{\mathbf{j}}, \Lambda_{-\mathbf{j}}\right)=K^{1} \frac{\Lambda_{j}^{1}}{\sum_{i} \Lambda_{i}^{1}}+K^{2} \frac{\Lambda_{j}^{2}}{\sum_{i} \Lambda_{i}^{2}}-\Gamma_{j}^{1} \Lambda_{j}^{1}-\Gamma_{j}^{2} \Lambda_{j}^{2}
$$

with $\left(\Gamma_{j}^{1}, \Gamma_{j}^{2}\right)=\left(\frac{\gamma_{j}^{1}-\gamma_{j}^{2} p_{21}}{1-p_{21} p_{12}}, \frac{\gamma_{j}^{2}-\gamma_{j}^{1} p_{12}}{1-p_{21} p_{12}}\right)$ for all $j$. We denote by $\left(\Lambda_{j}^{1 *}, \Lambda_{j}^{2 *}\right)$ the flow sent by source $j$ at equilibrium. We define a set $A$ :

$$
A:=\left\{(a, b) \in[0,1)^{2} \mid \forall j, \Lambda_{j}^{2 *} b-\Lambda_{j}^{1 *}>0 \text { or } \Lambda_{j}^{1 *} a-\Lambda_{j}^{2 *}>0\right\}
$$

In the first proposition we give an other form of (3) in order to compute the equilibrium.

Proposition 6. If $\left(p_{12}, p_{21}\right) \in A(H 1)$, then the game (3) is equivalent to the game (4) with $\left(\Lambda_{j}^{1}, \Lambda_{j}^{2}\right)=\left(\lambda_{j}^{1}+p_{21} \lambda_{j}^{2}, \lambda_{j}^{2}+p_{12} \lambda_{j}^{1}\right)$. 
Here, we define some new hypothesis.

$$
(H 2)\left\{\begin{array}{l}
\left\{\Gamma_{i}^{1}\right\}_{i} \text { are such that } \sum_{i} \Gamma_{i}^{1}-\max _{i}\left\{\Gamma_{i}^{1}\right\}(J-1)>0 \\
\left\{\Gamma_{i}^{2}\right\}_{i} \text { are such that } \sum_{i} \Gamma_{i}^{2}-\max _{i}\left\{\Gamma_{i}^{2}\right\}(J-1)>0
\end{array}\right.
$$

The next theorem gives us an explicit form of the equilibrium of the game when all sources send messages.

Theorem 7. Assume that (H1) and (H2) are satisfied. In this case the Nash Equilibrium is uniquely defined by:

$$
\left(\lambda_{j}^{1 *}, \lambda_{j}^{2 *}\right)= \begin{cases}\left(a_{j}^{1}, 0\right) & \text { if } \Lambda_{j}^{1 *}<\Lambda_{j}^{2 *} \\ \left(0, a_{j}^{2}\right) & \text { if } \Lambda_{j}^{1 *}>\Lambda_{j}^{2 *}\end{cases}
$$

with

$$
\begin{array}{cc}
a_{j}^{1}= & p_{21} \frac{(J-1) K^{2}}{\sum_{i} \gamma_{i}^{2}-\gamma_{i}^{1} p_{12}}\left(1-\frac{(J-1)\left(\gamma_{j}^{2}-\gamma_{j}^{1} p_{12}\right)}{\sum_{i} \gamma_{i}^{2}-\gamma_{i}^{1} p_{12}}\right) \\
& -\frac{(J-1) K^{1}}{\sum_{i} \gamma_{i}^{1}-\gamma_{i}^{2} p_{21}}\left(1-\frac{(J-1)\left(\gamma_{j}^{1}-\gamma_{j}^{2} p_{21}\right)}{\sum_{i} \gamma_{i}^{1}-\gamma_{i}^{2} p_{21}}\right) \\
a_{j}^{2}= & p_{12} \frac{(J-1) K^{1}}{\sum_{i} \gamma_{i}^{1}-\gamma_{i}^{2} p_{21}}\left(1-\frac{(J-1)\left(\gamma_{j}^{1}-\gamma_{j}^{2} p_{21}\right)}{\sum_{i} \gamma_{i}^{1}-\gamma_{i}^{2} p_{21}}\right) \\
& -\frac{(J-1) K^{2}}{\sum_{i} \gamma_{i}^{2}-\gamma_{i}^{1} p_{12}}\left(1-\frac{(J-1)\left(\gamma_{j}^{2}-\gamma_{j}^{1} p_{12}\right)}{\sum_{i} \gamma_{i}^{2}-\gamma_{i}^{1} p_{12}}\right) \\
\Lambda_{j}^{1 *}= & \frac{(J-1) K^{2}\left(p_{12} p_{21}-1\right)}{\sum_{i} \gamma_{i}^{2}-\gamma_{i}^{1} p_{12}}\left(1-\frac{(J-1)\left(\gamma_{j}^{2}-\gamma_{j}^{1} p_{12}\right)}{\sum_{i} \gamma_{i}^{2}-\gamma_{i}^{1} p_{12}}\right) \\
\Lambda_{j}^{2 *}= & \frac{(J-1) K^{1}\left(p_{12} p_{21}-1\right)}{\sum_{i} \gamma_{i}^{1}-\gamma_{i}^{2} p_{21}}\left(1-\frac{(J-1)\left(\gamma_{j}^{1}-\gamma_{j}^{2} p_{21}\right)}{\sum_{i} \gamma_{i}^{1}-\gamma_{i}^{2} p_{21}}\right)
\end{array}
$$

The previous theorem shows that under condition $(H 1)$ and $(H 2)$ all the sources send messages. And another important result is that each source just sends messages on one timeline. In the next section we study the effect of the parameter on the equilibrium.

\section{$6 \quad$ Numerical Illustrations}

We make our numerical illustrations with $\gamma_{j}^{1}=\gamma_{j}^{2}=\gamma$ for all $j$. We start fig. 1. at $p_{12}=0.75$ because with our parameters, when $p_{12}<0.75$ the condition $(H 1)$ is not verified. We see that $\lambda_{j}^{1 *}$ increasing in $p_{12}$ and $p_{21}$. In fig. 2. we can observe that sources (because symmetric sources have a symmetric behavior) prefer to send messages in timeline 2 when $p_{12}<0.4$ and $p_{21}=0.45$ or when $p_{12}<0.7$ and $p_{21}=0.7$. And they prefer to send in 


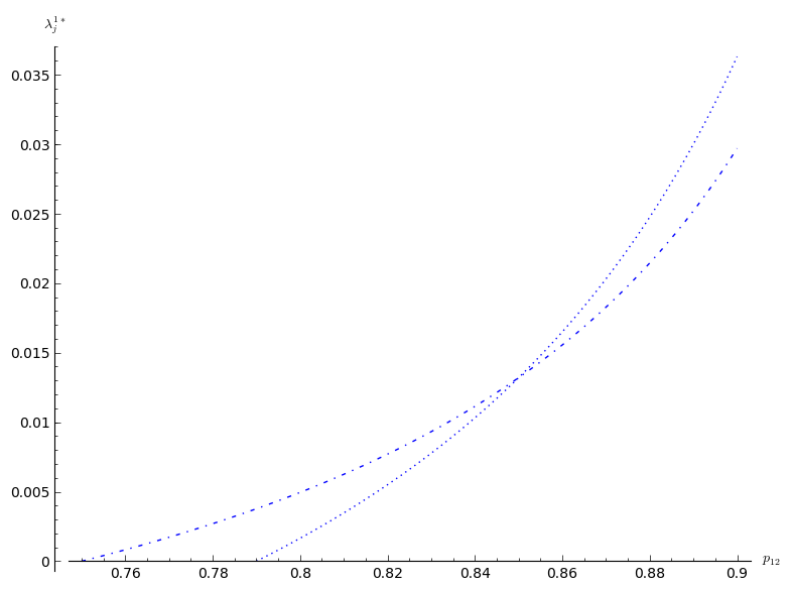

Figure 1: Behavior of $\lambda_{j}^{1 *}$ under $p_{12}$ with $\gamma=10, K^{1}=K^{2}=10, J=100$, $p_{21}=0.45$ for - . and $p_{21}=0.7$ for $\ldots$

timeline 1 in the other case. This can been seen when $p_{12}$ increases there is a preference for sending message in timeline 1 and when $p_{21}$ increases there is a preference for sending message in timeline 2 .

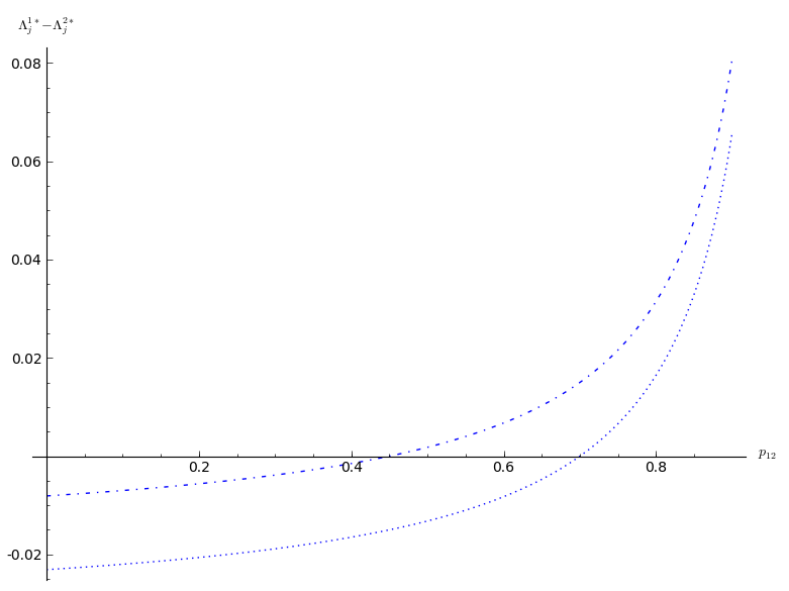

Figure 2: Behavior of $\Lambda_{j}^{1 *}-\Lambda_{j}^{2 *}$ under $p_{12}$ with $\gamma=10, K^{1}=K^{2}=10$, $J=100, p_{21}=0.45$ for - . and $p_{21}=0.7$ for $\ldots$ 


\section{Conclusions}

In this paper, we model a competition between information sources that use a social network timeline in order to disseminate and to maximize its visibility, particularly the visibility of its messages inside a timeline. We take into consideration that the messages in a timeline are of interest to the subscribers only if the messages are in the first $K$ positions (more $K$ recent). We have modeled the competition between sources using a noncooperative game and we have proved that this non-cooperative game is a standard game well known in telecommunication which has been studied in different contexts (resource sharing, communication, etc). We have also considered an interesting feature taking into account several timelines and the possibility for a message to be copied and relayed from one timeline to another. Then we have generalized our result and prove the existence of the equilibrium. In future works we plan to analyze more complex networks of timelines in which messages can be propagated between several timelines depending on the behavior of the timeline subscribers.

\section{References}

[1] E. Altman, "Game theoretic approaches for studying competition over popularity and over advertisement space in social networks", Valuetools, 2012.

[2] R. T. Ma, D. M. Chiu, J. C. Lui, V. Misra, D. Rubenstein, " On resource management for cloud users: A generalized kelly mechanism approach", Electrical Engineering, Tech. Rep., (2010).

[3] J. Ramesh,J . N. Tsitsiklis. "A game theoretic view of efficiency loss in resource allocation." Advances in Control, Communication Networks, and Transportation Systems. Birkhuser Boston, 2005. 203-223.

[4] Baccelli, F., Brmaud, P. (2003). Elements of queueing theory: Palm Martingale calculus and stochastic recurrences (Vol. 26). springer.

[5] Sina?, I. G. (1976). Introduction to ergodic theory (Vol. 18). Princeton University Press. 
[6] Tullock, G. (2001). Efficient rent seeking. In Efficient Rent-Seeking (pp. 3-16). Springer US.

[7] Chu, S. C. (2011). Viral advertising in social media: Participation in Facebook timelines and responses among college-aged users. Journal of Interactive Advertising, 12(1), 30-43.

[8] Kushin, M. J., Kitchener, K. (2009). Getting political on social network sites: Exploring online political discourse on Facebook. First Monday, $14(11)$.

[9] Small, T. (2008). The facebook effect? on-line campaigning in the 2008 canadian and us elections. POLICY, 85.

[10] Tucker, C. (2012). Social advertising. Available at SSRN 1975897.

[11] Monderer, D., Shapley, L. S. (1996). Potential games. Games and economic behavior, 14(1), 124-143.

[12] Altman, E., Kumar, P., Venkatramanan, S., Kumar, A. Competition Over Timeline in Social Networks. ASSONAM 2013

[13] Baccelli, F., Brmaud, P. (2003). Elements of queueing theory: Palm Martingale calculus and stochastic recurrences (Vol. 26). springer.

[14] Nisan, N. (Ed.). (2007). Algorithmic game theory. Cambridge University Press.

[15] Vazirani, V. V. (2007). Algorithmic game theory (Vol. 1). T. Roughgarden, \& E. Tardos (Eds.). Cambridge: Cambridge University Press.

\footnotetext{
APPENDIX

Proof of proposition 2

If all sources $\{1, \cdots, J\}-\{j\}$ play $0, j$ can always find a very small $\lambda_{j}^{*}$ such that $\lambda_{j}^{*}<\frac{K}{\gamma_{j}}$. Then, we have that $U_{j}\left(\lambda_{j}^{*}, 0\right)>0$ and the decision vector $(0, \ldots, 0)$ is not a Nash equilibrium.
}

\section{Proof of theorem 3}

The first order condition:

$$
\frac{\partial U_{j}}{\lambda_{j}}\left(\lambda_{j}, \lambda_{-\mathbf{j}}\right)=0
$$


give us the best reply of the player $j$.

$$
\begin{aligned}
& \frac{\partial U_{j}}{\lambda_{j}}\left(\lambda_{j}, \lambda_{-\mathbf{j}}\right)=0 \\
& \Leftrightarrow\left(\frac{1}{\sum_{i} \lambda_{i}}-\frac{\lambda_{j}}{\left(\sum_{i} \lambda_{i}\right)^{2}}\right)=\frac{\gamma_{j}}{K} \\
& \Leftrightarrow\left(\frac{\sum_{i} \lambda_{i}-\lambda_{j}}{\left(\sum_{i} \lambda_{i}\right)^{2}}\right)=\frac{\gamma_{j}}{K} .(*)
\end{aligned}
$$

In the last equation, by taking the sum over $j$, we have:

$$
\begin{gathered}
J\left(\sum_{i} \lambda_{i}\right)-\frac{1}{K} \sum_{i} \gamma_{i}\left(\sum_{i} \lambda_{i}\right)^{2}=\left(\sum_{i} \lambda_{i}\right) \\
\Leftrightarrow(J-1)\left(\sum_{i} \lambda_{i}\right)-\frac{1}{K} \sum_{i} \gamma_{i}\left(\sum_{i} \lambda_{i}\right)^{2}=0 \\
\Leftrightarrow\left(\sum_{i} \lambda_{i}\right)\left((J-1)-\frac{1}{K} \sum_{i} \gamma_{i}\left(\sum_{i} \lambda_{i}\right)\right)=0 \\
\Leftrightarrow \sum_{i} \lambda_{i}=\frac{(J-1) K}{\sum_{i} \gamma_{i}} .
\end{gathered}
$$

The closed form expression is obtained by using (8) and (*). The unicity comes from that $\lambda_{j}^{*}$ is strictly defined by $\sum_{i} \lambda_{i}^{*}$ and $\sum_{i} \lambda_{i}^{*}$ is unique.

\section{Proof of theorem 4}

We only have to prove that if $j^{\prime}$ plays 0 at equilibrium, all the other sources $j<j^{\prime}$ play also 0 . This fact is clear because $\lambda_{j}^{*}$ is decreasing in $\gamma_{j}$.

Proof of theorem 5

We have to compute $U_{j}\left(\lambda_{j}^{*}, \lambda_{-j}{ }^{*}\right)$.

$$
\begin{aligned}
U_{j}\left(\lambda_{j}^{*}, \lambda_{-j}{ }^{*}\right) & =\left(1-\frac{(J-1) \gamma_{j}}{\sum_{i} \gamma_{i}}\right)-\gamma_{j}\left(\frac{(J-1) K}{\sum_{i} \gamma_{i}}\right)\left(1-\frac{(J-1) \gamma_{j}}{\sum_{i} \gamma_{i}}\right) \\
& =\left(1-\frac{(J-1) \gamma_{j}}{\sum_{i} \gamma_{i}}\right)\left(1-\gamma_{j} \frac{(J-1) K}{\sum_{i} \gamma_{i}}\right) \\
& =\frac{1}{\sum_{i} \gamma_{i}}\left(\sum_{i} \gamma_{i}-(J-1) \gamma_{j}\right)\left(\sum_{i} \gamma_{i}-\gamma_{j}(J-1) K\right)
\end{aligned}
$$

And we can deduce the form of the price of anarchy which is:

$$
P_{o} A=\frac{\left(\sum_{i} \gamma_{i}\right)\left(K-\underline{\lambda} \sum_{i} \gamma_{i}\right)}{\sum_{j}\left(\sum_{i} \gamma_{i}-(J-1) \gamma_{j}\right)\left(\sum_{i} \gamma_{i}-\gamma_{j}(J-1) K\right)}
$$

\section{Proof of proposition 6}

We are going to prove this theorem in two steps.

Step 1: let us look at the limit of the average number of messages in the two timelines for player $j$ (i.e the first part of the objective function of the player $j$ ). We can notice that

$$
\begin{aligned}
& K^{1} \frac{\lambda_{j}^{1}+p_{21} \lambda_{j}^{2}}{\sum_{i}\left(\lambda_{i}^{1}+p_{21} \lambda_{i}^{2}\right)}+K^{2} \frac{\lambda_{j}^{2}+p_{12} \lambda_{j}^{1}}{\sum_{i}\left(\lambda_{i}^{2}+p_{12} \lambda_{i}^{1}\right)} \\
& =K^{1} \frac{\Lambda_{j}^{1}}{\sum_{i} \Lambda_{i}^{1}}+K^{2} \frac{\Lambda_{j}^{2}}{\sum_{i} \Lambda_{i}^{2}}
\end{aligned}
$$


with $\Lambda_{j}^{1}=\lambda_{j}^{1}+p_{21} \lambda_{j}^{2}$ and $\Lambda_{j}^{2}=\lambda_{j}^{2}+p_{12} \lambda_{j}^{1}$ for all $j$.

Step 2: We want to find $\Gamma_{j}^{1}$ and $\Gamma_{j}^{2}$ such that $\Gamma_{j}^{1} \Lambda_{j}^{1}+\Gamma_{j}^{2} \Lambda_{j}^{2}=\gamma_{j}^{1} \lambda_{j}^{1}+\gamma_{j}^{2} \lambda_{j}^{2}$. Firstly $\left(\lambda_{j}^{1}, \lambda_{j}^{2}\right)=\left(\frac{\Lambda_{j}^{2} p_{21}-\Lambda_{j}^{1}}{p_{12} p_{21}-1}, \frac{\Lambda_{j}^{1} p_{12}-\Lambda_{j}^{2}}{p_{12} p_{21}-1}\right)$ is solution of

$$
\left\{\begin{array}{l}
\Lambda_{j}^{1}=\lambda_{j}^{1}+p_{21} \lambda_{j}^{2} \\
\Lambda_{j}^{2}=\lambda_{j}^{2}+p_{12} \lambda_{j}^{1}
\end{array}\right.
$$

Secondly, with the above result we can calculate $\Gamma_{j}^{1}$ and $\Gamma_{j}^{2}$. In fact,

$$
\gamma_{j}^{1} \lambda_{j}^{1}+\gamma_{j}^{2} \lambda_{j}^{2}=\frac{1}{p_{12} p_{21}-1}\left[\Lambda_{j}^{1}\left(\gamma_{j}^{2} p_{12}-\gamma_{j}^{1}\right)+\Lambda_{j}^{2}\left(\gamma_{j}^{1} p_{21}-\gamma_{j}^{2}\right)\right] \text {. }
$$

Thus we can conclude that

$$
\left(\Gamma_{j}^{1}, \Gamma_{j}^{2}\right)=\left(\frac{\gamma_{j}^{2} p_{12}-\gamma_{j}^{1}}{p_{12} p_{21}-1}, \frac{\gamma_{j}^{1} p_{21}-\gamma_{j}^{2}}{p_{12} p_{21}-1}\right) .
$$

\section{Proof of theorem 7}

The proof follows two steps. Firstly, we calculate the equilibrium of (4) and secondly we make the change of variables in order to have the value of the direct flow of each sources at equilibrium.

Step 1: In order to find the equilibrium of (4) we have to compute the best reply. We do it just for one $\Lambda_{j}^{1}$. It works the same way as $\Lambda_{j}^{1}$ for all the other $j$ and timeline 2 .

$$
\begin{array}{ll}
\frac{\partial U_{j}}{\partial \Lambda_{j}^{1}}\left(\boldsymbol{\Lambda}_{\mathbf{j}}, \boldsymbol{\Lambda}_{-\mathbf{j}}\right) & =0 \\
K^{1}\left(\frac{1}{\sum_{i} \Lambda_{i}^{1}}-\frac{\Lambda_{j}^{1}}{\left(\sum_{i} \Lambda_{i}^{1}\right)^{2}}\right)-\Gamma_{j}^{1} & =0
\end{array}
$$

We remark that the best reply just depend of $\left\{\lambda_{j}^{1}\right\}_{j}$ and it is equivalent to the best reply of the game (2) where the objectif function for each player $j$ is:

$$
\max _{\Lambda_{j}^{1}} U_{j}\left(\boldsymbol{\Lambda}_{\mathbf{j}}, \Lambda_{-\mathbf{j}}\right)=K^{1} \frac{\Lambda_{j}^{1}}{\sum_{i} \Lambda_{i}^{1}}-\Gamma_{j}^{1} \Lambda_{j}^{1}
$$

This is why by using theorem 3 we have

$$
\forall j,\left(\Lambda_{j}^{1 *}, \Lambda_{j}^{2 *}\right)=\left(\frac{(J-1) K^{1}}{\sum_{i} \Gamma_{i}^{1}}\left(1-\frac{\Phi^{1} \Gamma_{j}^{1}}{K^{1}}\right), \frac{(J-1) K^{2}}{\sum_{i} \Gamma_{i}^{2}}\left(1-\frac{\Phi^{2} \Gamma_{j}^{2}}{K^{2}}\right)\right)
$$

with $\left(\Phi^{1}, \Phi^{2}\right)=\left(\frac{(J-1) K^{1}}{\sum_{i} \Gamma_{i}^{1}}, \frac{(J-1) K^{2}}{\sum_{i} \Gamma_{i}^{2}}\right)$.

Step 2: In the proof of proposition 10 we have proved that $\left(\lambda_{j}^{1}, \lambda_{j}^{2}\right)=$ $\left(\frac{\Lambda_{j}^{2} p_{21}-\Lambda_{j}^{1}}{p_{12} p_{21}-1}, \frac{\Lambda_{j}^{1} p_{12}-\Lambda_{j}^{2}}{p_{12} p_{21}-1}\right)$. This is why by using this fact we have: 


$$
\begin{aligned}
\lambda_{j}^{1}= & p_{21} \frac{(J-1) K^{2}}{\sum_{i} \gamma_{i}^{2}-\gamma_{i}^{1} p_{12}}\left(1-\frac{(J-1)\left(\gamma_{j}^{2}-\gamma_{j}^{1} p_{12}\right)}{\sum_{i} \gamma_{i}^{2}-\gamma_{i}^{1} p_{12}}\right) \\
& -\frac{(J-1) K^{1}}{\sum_{i} \gamma_{i}^{1}-\gamma_{i}^{2} p_{21}}\left(1-\frac{(J-1)\left(\gamma_{j}^{1}-\gamma_{j}^{2} p_{21}\right)}{\sum_{i} \gamma_{i}^{1}-\gamma_{i}^{2} p_{21}}\right) \\
\lambda_{j}^{2}= & p_{12} \frac{(J-1) K^{1}}{\sum_{i} \gamma_{i}^{1}-\gamma_{i}^{2} p_{21}}\left(1-\frac{(J-1)\left(\gamma_{j}^{1}-\gamma_{j}^{2} p_{21}\right)}{\sum_{i} \gamma_{i}^{1}-\gamma_{i}^{2} p_{21}}\right) \\
& -\frac{(J-1) K^{2}}{\sum_{i} \gamma_{i}^{2}-\gamma_{i}^{1} p_{12}}\left(1-\frac{(J-1)\left(\gamma_{j}^{2}-\gamma_{j}^{1} p_{12}\right)}{\sum_{i} \gamma_{i}^{2}-\gamma_{i}^{1} p_{12}}\right)
\end{aligned}
$$

Now in $(H 1)$ we have assume that $\Lambda_{j}^{2 *} p_{21}-\Lambda_{j}^{1 *}>0$ or $\Lambda_{j}^{1 *} p_{12}-\Lambda_{j}^{2 *}>0$. We notice that if $\Lambda_{j}^{2 *}>\Lambda_{j}^{1 *}$ then $\Lambda_{j}^{1 *} p_{12}-\Lambda_{j}^{2 *}<0$ which implies that $\lambda_{j}^{2 *}=0$. And if $\Lambda_{j}^{2 *}<\Lambda_{j}^{1 *}$ then $\Lambda_{j}^{2 *} p_{21}-\Lambda_{j}^{1 *}<0$ which implies that $\lambda_{j}^{1 *}=0$. This is why a player sends messages just on one wall. 